\title{
Efecto del pretratamiento térmico de residuos de alimentos sobre la producción de metano
}

\section{Effect of the thermal pretreatment of from food waste on methane production}

\author{
Brayan Alexis Parra-Orobio ${ }^{\text {; }}$ Carlos Vásquez-Franco²; Wilmar Alexander Torres-López ; \\ Luis Fernando Marmolejo-Rebellón ${ }^{4}$; Patricia Torres-Lozada ${ }^{5}$
}

\begin{abstract}
${ }^{1}$ Ing. Sanitario. M.Sc. Universidad del Valle, Grupo de Investigación Estudio y Control de la Contaminación Ambiental-ECCA. Cali, Colombia, e-mail: brayan. parra@correounivalle.edu.co, Dhttps://orcid.org/0000-0001-9256-6797
\end{abstract}

${ }^{2}$ Ing. Sanitario. Universidad del Valle, Grupo de Investigación Estudio y Control de la Contaminación Ambiental-ECCA. Cali, Colombia, e-mail: carlos. vasquez@correounivalle.edu.co, (1)https://orcid.org/0000-0001-9798-5077

${ }^{3}$ Estadístico. Universidad del Valle, Departamento de Biología. Cali, Colombia, e-mail: wilmar.alexander.torres@correounivalle.edu.co, (Dhttps://orcid. org/0000-0002-2482-013X

${ }^{4}$ Ing. Sanitario. M.Sc. PhD. Universidad del Valle, Grupo de Investigación Estudio y Control de la Contaminación Ambiental-ECCA. Cali, Colombia, e-mail: Iuis. marmolejo@correounivalle.edu.co, Dhttps://orcid.org/0000-0001-9993-2841

${ }^{5}$ Ing. Sanitaria. M.Sc. PhD. Universidad del Valle, Grupo de Investigación Estudio y Control de la Contaminación Ambiental-ECCA. Cali, Colombia, e-mail: patricia.torres@correounivalle.edu.co, Dhttps://orcid.org/0000-0001-9323-6677

Cómo citar: Parra-Orobio, B.A.; Vásquez-Franco, C.; Torres-López, W.A.; Marmolejo-Rebellón, L.F.; Torres-Lozada, P. 2019. Efecto del pretratamiento térmico de residuos de alimentos sobre la producción de metano. Rev. U.D.C.A Act. \& Div. Cient. 22(1):e1220. https:// doi.org/10.31910/rudca.v22.n1.2019.1220

Artículo de acceso abierto publicado por Revista U.D.C.A Actualidad \& Divulgación Científica bajo una licencia Creative Commons CC BY-NC 4.0

Recibido: Agosto 17 de 2018

Aceptado: Mayo 15 de 2019

\section{RESUMEN}

Los residuos de alimentos son el componente principal de los residuos sólidos municipales y dado su elevado contenido de materia orgánica tienen alto potencial de producción de metano, mediante la digestión anaerobia; sin embargo, la predominancia de material lignocelulósico dificulta su hidrólisis. En este estudio, mediante ensayos de potencial bioquímico de metano, se evaluó el efecto de diferentes condiciones de pretratamiento térmico del sustrato, aplicando temperaturas entre $72-128^{\circ} \mathrm{C}$ y tiempos de exposición de 15 - 33 minutos sobre el rendimiento de producción de $\mathrm{CH}_{4}$. Para evaluar el efecto de las condiciones de pretratamiento, se empleó la metodología de superficie de respuesta y la aplicación de los modelos cinéticos de primer orden y de ajuste de Gompertz modificado. Los parámetros cinéticos identificados fueron validados, mediante niveles de confianza, usando la matriz de información de fisher. Se encontró que la región óptima, para alcanzar un mayor rendimiento en la digestión anaerobia, en cuanto a la producción de $\mathrm{CH}_{4}$, superior a $150 \mathrm{mLCH}_{4} \mathrm{gSV}^{1}$ y tiempos de la fase de latencia menores a 1 día, fue alrededor de $100^{\circ} \mathrm{C}$, con tiempos de exposición cercanos a 15 minutos, condición en que se alcanzó una mayor solubilidad y mejorando positivamente la etapa hidrolítica del proceso anaerobio.

Palabras clave: cinética; digestión anaerobia; metano; residuos sólidos; temperatura. 
Parra-Orobio, B.A.; Vásquez-Franco, C.; Torres-López, W.A.; Marmolejo-Rebellón, L.F.; Torres-Lozada, P.: Pretratamiento en producción de metano

\section{ABSTRACT}

Food waste are the main component of the municipal solid waste and given their high content of organic matter, have high potential for methane production through the anaerobic digestion. However, the predominance of lignocellulosic material hinders its hydrolysis. In this study, by means of assays of biochemical methane potential, the effect of different thermal pre-treatment conditions of the substrate was evaluated by applying temperatures between 72 $128^{\circ} \mathrm{C}$ and exposure times of $15-33$ minutes on the conditions on the performance of $\mathrm{CH}_{4}$. To evaluate the effect of pretreatment, we used the Response Surface Methodology accompanied by the application of first order kinetic and modified Gompertz models. The identified kinetic parameters were validated using confidence levels using the fisher information matrix. It was found that the optimal region, to reach a higher yield in the anaerobic digestion with regard to the production of $\mathrm{CH}_{4}$ higher than $150 \mathrm{mLCH}_{4}$ $\mathrm{gVS}^{-1}$ and lag-phase times less than $1 \mathrm{day}$, was around $100^{\circ} \mathrm{C}$ with exposure times close to 15 minutes, condition in which a greater solubility was reached and positively improving the hydrolytic stage of the anaerobic process.

Keywords: anaerobic digestion; kinetic; methane; solid waste; temperature.

\section{INTRODUCCIÓN}

A través de los años, la cantidad de residuos sólidos municipalesRSM ha ido en aumento, debido al desarrollo económico y al incremento de la población mundial. Los residuos de alimentos-RA constituyen el 40\% de los RSM en países desarrollados y del orden del 75\%, en los países en desarrollo (Cabeza et al. 2016), con cifras de 1.300 millones de toneladas, en países desarrollados (Dhamodharan et al. 2015) y 160 en países en desarrollo, particularmente, en Latinoamérica y el Caribe - LAC (Sepúlveda, 2016). Estos residuos presentan un alto contenido de humedad, entre el 70 y $90 \%$, relaciones SV/ST superiores al 80\% y C/N entre 15 y 36 (Zhang et al. 2014; Thi et al. 2015).

La digestión anaerobia-DA es una alternativa tecnológica, que consiste en la conversión de la materia orgánica presente en el sustrato, en biogás y se lleva a cabo por un grupo de microorganismos, a través diferentes de etapas metabólicas: hidrólisis, acidogénesis, acetogénesis y metanogénesis (Angelidaki et al. 2009). La composición de biogás que se obtiene varía dependiendo del tipo de materia prima $\mathrm{y}$ de las condiciones de funcionamiento del digestor (Surendra et al. 2014); el biogás, se compone de 50 a 75\% de $\mathrm{CH}_{4}$ y 25 a 50\% de $\mathrm{CO}_{2}$, junto con otros componentes, traza como vapor de agua $\left(\mathrm{H}_{2} \mathrm{O}\right)$, sulfuro de hidrógeno $\left(\mathrm{H}_{2} \mathrm{~S}\right)$ y amoníaco $\left(\mathrm{NH}_{3}\right)$.

En general, la hidrólisis, se presenta como la etapa limitante en la degradación de sustratos orgánicos heterogéneos y complejos, como los RA (Ma et al. 2018; Raposo et al. 2012). En esta etapa, se pueden formar subproductos tóxicos, como compuestos heterocíclicos y ácidos grasos volátiles, que alteran los grupos microbianos metanogénicos y, por ende, la calidad del biogás (Neves et al. 2006). Diferentes estrategias de pretratamiento del sustrato, se han implementado, para maximizar la producción de metano $\left(\mathrm{CH}_{4}\right)$, a partir de la DA, como procesos mecánicos, químicos, biológicos y térmicos, todos con el fin de la solubilizar y liberar macromoléculas de la materia orgánica, que mejoren el rendimiento del proceso (Kempegowda et al. 2017; Parthiba Karthikeyan et al. 2018).

Dentro de los estudios enfocados al pretratamiento térmico, se encuentran los realizados por Liu et al. (2012), quienes determinaron que el tratamiento térmico a RA, a $175^{\circ} \mathrm{C}$, disminuye hasta un $11,7 \%$ de la producción de metano; esto lo atribuyeron a la formación de melanoidinas. Por su parte, Ariunbaatar et al. (2014) encontraron que el pretratamiento de RA, a temperaturas entre 50 y $80^{\circ} \mathrm{C}$, por periodos de tiempo entre 1,5 y 12 horas, mejoran la producción de metano, en un 40\%. Li et al. (2016) obtuvieron un incremento en la producción de biogás del 69 y 75\%, en pretratamientos, cuya duración fue de $70 \mathrm{~min}-90^{\circ} \mathrm{C}$ y $50 \mathrm{~min}-120^{\circ} \mathrm{C}$, respectivamente; sin embargo, pocos estudios abordan la incidencia del pretratamiento desde una perspectiva de la cinética del proceso (Ma et al. 2018).

Por lo anterior, este estudio evaluó, mediante ensayos de Potencial Bioquímico de Metano-PBM, el efecto de pretratamiento térmico en los residuos de alimentos sobre la producción de metano. Se evaluó su incidencia sobre la hidrólisis del proceso, considerando aspectos clave del proceso, como el $\mathrm{pH}$, la alcalinidad bicarbonática y los ácidos grasos volátiles, empleando herramientas de la cinética y la metodología de superficie de respuesta-MSR.

\section{MATERIALES Y MÉTODOS}

Sustrato e inóculo. El sustrato, se tomó de un restaurante universitario de la ciudad de Cali - Colombia, que atiende a más de 3.000 estudiantes al día y genera $86,6 \mathrm{~kg} \cdot \mathrm{d}^{-1}$, que representan la composición típica de una población de 15.000 habitantes, correspondiendo, en un 56\%, a carbohidratos, asociado a cáscaras de plátano, papa, entre otros; $25 \%$, a frutas cítricas; $8,2 \%$, a frutas no cítricas; $8,2 \%$, a fibras y minerales, relacionado a cáscaras de huevo, apio, hierbas, entre otros; además, un 3,2\%, de hierbas, atribuido a cilantro, acelgas, cidra y otros. Esta composición fue establecida de acuerdo con lo encontrado por Oviedo-Ocaña et al. (2014) y SotoPaz et al. (2019), en los RSM, de una localidad que realiza recolección selectiva y separación en la fuente de sus residuos.

Como inóculo, se utilizó biomasa anaerobia activa, procedente del digestor de lodos de una PTAR municipal, que cuenta con tratamiento primario avanzado y trata el agua residual de una población de 2 millones de habitantes (Parra-Orobio et al. 2018). En la tabla 1, se presentan las características fisicoquímicas del sustrato y el inóculo, que fueron caracterizados de acuerdo con ICONTEC (2004), ICONTEC (2009) y APHA (2005).

Pretratamiento del sustrato. Previamente a los ensayos PBM, los RA fueron sometidos a un pretratamiento mecánico, utilizando una trituradora CB15 Waring Commercial, a una velocidad de 15800rpm, 
Tabla 1. Caracterización fisicoquímica de los residuos de alimentos e inóculo.

\begin{tabular}{|c|c|c|c|}
\hline Parámetros & RA & Inóculo $^{\mathrm{m}}$ & Unidades \\
\hline $\mathrm{pH}(\mathrm{n}=8)$ & $5,6 \pm 0.1$ & $7,9 \pm$ & Unidades \\
\hline Humedad $(\mathrm{n}=8)$ & $76 \pm 0.9$ & N.D & $\%$ \\
\hline $\operatorname{AT}(\mathrm{n}=8)$ & $4021 \pm 247$ & 35862.2 & $\mathrm{mg} \mathrm{CaCO}_{3} \cdot \mathrm{L}^{-1}$ \\
\hline $\mathrm{AB}(\mathrm{n}=8)$ & $0 \pm 0$ & $2525.2 \pm$ & $\mathrm{mg} \mathrm{CaCO}_{3} \cdot \mathrm{L}^{-1}$ \\
\hline $\mathrm{AB} / \mathrm{AT}$ & 0 & 0.71 & \\
\hline$A G V^{\prime} s(n=8)$ & $3650 \pm 235$ & $1071 \pm$ & $\mathrm{mg} \cdot \mathrm{L}^{-1}$ \\
\hline DQO ${ }_{\text {total }}(\mathrm{n}=8)$ & $112522 \pm 410$ & N.D & $\mathrm{mg} \cdot \mathrm{L}^{-1}$ \\
\hline $\mathrm{DQO}_{\text {filtrada }}(\mathrm{n}=8)$ & $22667 \pm 342$ & N.D & $\mathrm{mg} \cdot \mathrm{L}^{-1}$ \\
\hline $\mathrm{DBO}_{5}(\mathrm{n}=8)$ & $66951 \pm 10$ & N.D & $\mathrm{mg} \cdot \mathrm{L}^{-1}$ \\
\hline $\mathrm{DBO}_{5} / \mathrm{DQO}$ & 0.59 & N.D & - \\
\hline $\mathrm{ST}(\mathrm{n}=8)$ & $110730 \pm 488$ & 35263,3 & $\mathrm{mg} \cdot \mathrm{L}^{-1}$ \\
\hline $\mathrm{SV}(\mathrm{n}=8)$ & $93410 \pm 331$ & 15826,3 & $\mathrm{mg} \cdot \mathrm{L}^{-1}$ \\
\hline $\mathrm{SV} / \mathrm{ST}$ & 0,8 & 0,4 & - \\
\hline Carbono Orgánico Total $(n=4)$ & $39850 \pm 3000$ & N.D & $\mathrm{mg} \cdot \mathrm{kg}^{-1}$ \\
\hline Nitrógeno Total $(\mathrm{n}=4)$ & $1186 \pm 201$ & N.D & $\mathrm{mg} \cdot \mathrm{kg}^{-1}$ \\
\hline Nitrógeno Amoniacal total(n=4) & $86 \pm 63$ & N.D & $\mathrm{mg} \cdot \mathrm{L}^{-1}$ \\
\hline Fósforo total $(\mathrm{n}=4)$ & $265 \pm 295$ & N.D & $\mathrm{mg} \cdot \mathrm{kg}^{-1}$ \\
\hline $\mathrm{C} / \mathrm{N}$ & 33,6 & N.D & - \\
\hline AME & N.D & 0,008 & $\mathrm{gDQO} \cdot \mathrm{gSV}^{1} \cdot \mathrm{d}^{-1}$ \\
\hline
\end{tabular}

m: corresponde a 3 muestras n: número de muestras

durante un minuto (velocidad estándar del equipo), garantizando tamaños de partículas inferiores a 30mm (Izumi et al. 2010; ParraOrobio et al. 2017).

El pretratamiento térmico del sustrato, se realizó en un horno WTB Binder 78532. La definición de las condiciones de pretratamiento, se efectuó con base en la literatura y en la metodología de Superficie de Respuesta (Parthiba Karthikeyan et al. 2018), empleándose un diseño experimental factorial $2^{2}$ con dos factores, los cuales, fueron temperatura y tiempo; cada uno, con dos niveles, respectivamente. El punto central fue $100^{\circ} \mathrm{C}$ y 22 minutos, que se definió de acuerdo con los resultados obtenidos por Li et al. (2016) y Parthiba Karthikeyan et al. (2018); en dicho punto, se efectuaron cinco réplicas, como lo requiere la MSR. Además, fueron planteados cuatro puntos axiales, conformados por temperaturas de $72,80,100$ y $128^{\circ} \mathrm{C}$ y tiempos de exposición de 12, 15,30 y 33 minutos, obtenidos aplicando la MSR y cada uno de éstos, se ejecutó por triplicado. Adicionalmente, se incluyó una unidad experimental, denominada control, en la que el sustrato no fue pretratado. La tabla 2 muestra el diseño experimental y codificado de los factores como $\mathrm{X}_{1}$ y $\mathrm{X}_{2}$.

Se construyó un modelo ajustado, representado mediante la Ecuación 1, con la finalidad de analizar el efecto de los factores temperatura y tiempo de exposición sobre de las variables de respuesta PBM, $\mathrm{pH}, \mathrm{AB}$ y $\mathrm{AGV}$ 's.

$$
y=b_{0}+b_{1} T+b_{2} t+b_{3} T * t+b_{4} T^{2}+b_{5} t^{2} \quad \text { Ecuación.1 }
$$

Donde:

$y$ : variable de respuesta analizada: $\mathrm{PBM}, \mathrm{pH}, \mathrm{AB}$ y AGV's.

$b_{0}$ : intercepto.

$b_{1}$ y $b_{2}$ : coeficientes que acompañan a las covariables temperatura y tiempo en términos lineales, respectivamente.

$b_{3}$ : coeficiente de interacción entre las covariables temperatura y tiempo.

$b_{4}$ y $b_{5}$ : coeficientes que acompañan a las covariables temperatura y tiempo en términos cuadráticos, respectivamente.

Ensayos de potencial bioquímico de metano-PBM. La cuantificación de biogás en cada una de las condiciones experimentales mostradas en la tabla 2, se realizó mediante el método manométrico, empleando el Sistema Oxitop®, con reactores de 250mL (Pabón et al. 2012). Los ensayos, se realizaron en una incubadora WTW TS 606-G/2-i, con agitación manual intermitente; el volumen útil empleado fue de $200 \mathrm{~mL}$. Para garantizar que la medición manométrica del biogás correspondiera predominantemente a metano, se capturó el dióxido de carbono, a través de perlas de $\mathrm{NaOH}$, cuya composición fue verificada mediante cromatografía de gases, usando el Cromatógrafo GC2014. El volumen de metano a condiciones estándar-CE, se determinó de acuerdo con Parra-Orobio et al. (2015), donde se considera la proporción de metano disuelto. 
Tabla 2. Diseño experimental para la optimización entre la temperatura y el tiempo de exposición.

\begin{tabular}{|c|c|c|c|c|}
\hline \multirow{2}{*}{ Tratamiento } & \multicolumn{2}{|c|}{ Variables Naturales } & \multicolumn{2}{c|}{ Variables Codificadas } \\
\cline { 2 - 5 } & Temperatura $\left({ }^{\circ} \mathbf{C}\right)$ & $\begin{array}{c}\text { Tiempo de ex- } \\
\text { posición (minutos) }\end{array}$ & $\mathbf{X}_{1}$ & $\mathbf{X}_{2}$ \\
\hline T1 & 80 & 15 & -1 & -1 \\
\hline T2 & 80 & 30 & -1 & 1 \\
\hline T3 & 120 & 15 & 1 & -1 \\
\hline T4 & 120 & 30 & 1 & 1 \\
\hline T5 & 100 & 22 & - & 0 \\
\hline T6 & 72 & 22 & 0 & 0 \\
\hline T7 & 128 & 22 & 0 & - \\
\hline T8 & 100 & 12 & - & - \\
\hline T9 & 100 & 33 & & \\
\hline T10 & Control & Sin tiempo & & 0 \\
\hline
\end{tabular}

La disponibilidad de nutrientes que se requiere en el ensayo de PBM fue suplida, de acuerdo con lo sugerido por Holliger et al. (2016). La cantidad utilizada de macro y de micronutrientes en cada uno de los reactores fue de $1 \mathrm{~mL}$ por cada litro útil del reactor. La solución de macronutrientes estaba constituida por $\mathrm{NH}_{4} \mathrm{Cl}\left(170 \mathrm{~g} \cdot \mathrm{L}^{-1}\right), \mathrm{NaHCO}_{3}$ $\left(1 \mathrm{~g} \mathrm{~L}^{-1}\right), \mathrm{KH}_{2} \mathrm{PO}_{4}\left(37 \mathrm{~g} \cdot \mathrm{L}^{-1}\right), \mathrm{MgSO}_{4} 4 \mathrm{H}_{2} \mathrm{O}\left(9 \mathrm{~g} \cdot \mathrm{L}^{-1}\right)$ y $\mathrm{CaCl}_{2} 2 \mathrm{H}_{2} \mathrm{O}$ $\left(8 \mathrm{~g} \cdot \mathrm{L}^{-1}\right)$, mientras que la de micronutrientes por $\mathrm{FeCl}_{2} 6 \mathrm{H}_{2} \mathrm{O}\left(2 \mathrm{~g} \cdot \mathrm{L}^{-1}\right)$, $\mathrm{ZnCl}_{2}\left(0,05 \mathrm{~g} \cdot \mathrm{L}^{-1}\right), \mathrm{CuCl}_{2} 2 \mathrm{H}_{2} \mathrm{O}\left(0,03 \mathrm{~g} \cdot \mathrm{L}^{-1}\right), \mathrm{MnCl}_{2} 4 \mathrm{H}_{2} \mathrm{O}\left(0,5 \mathrm{~g} \cdot \mathrm{L}^{-1}\right)$, $\left(\mathrm{NH}_{4}\right) \mathrm{Mo}_{7} \mathrm{O}_{24} 4 \mathrm{H}_{2} \mathrm{O}\left(0,09 \mathrm{~g} \cdot \mathrm{L}^{-1}\right), \mathrm{AlCl}_{3} 6 \mathrm{H}_{2} \mathrm{O}\left(0,05 \mathrm{~g} \cdot \mathrm{L}^{-1}\right), \mathrm{CoCl}_{2} 6 \mathrm{H}_{2} \mathrm{O}$ $\left(2 \mathrm{~g} \cdot \mathrm{L}^{-1}\right), \mathrm{NiCl}_{2} 6 \mathrm{H}_{2} \mathrm{O}\left(0,05 \mathrm{~g} \cdot \mathrm{L}^{-1}\right), \mathrm{H}_{3} \mathrm{BO}_{3}\left(0,05 \mathrm{~g} \cdot \mathrm{L}^{-1}\right), \mathrm{Na}_{2} \mathrm{SeO}_{3} 5 \mathrm{H}_{2} \mathrm{O}$ $\left(0,1 \mathrm{~g} \mathrm{~L}^{-1}\right)$, EDTA $\left(1 \mathrm{~g} \cdot \mathrm{L}^{-1}\right)$ y $\mathrm{HCl}\left(1 \mathrm{~mL} \cdot \mathrm{L}^{-1}\right)$. Para garantizar un ambiente totalmente Redox, se adicionó en cada uno de los reactores Resarzurina $\left(0,5 \mathrm{~g} \cdot \mathrm{L}^{-1}\right)$ y $\mathrm{NaS} 7 \mathrm{H}_{2} \mathrm{O}\left(0,1 \mathrm{~g} \cdot \mathrm{L}^{-1}\right.$ ) (Aquino et al. 2007; Angelidaki et al. 2009; Torres \& Pérez, 2010).

El pH, se acondicionó a 7,0 unidades, con una solución de $\mathrm{NaHCO}_{3}$, al $4 \%$ v/v y se mantuvo una temperatura de $35^{\circ} \mathrm{C}$, durante 63 días. La relación sustrato-inóculo-S/I empleada fue de $1,0 \mathrm{gSV}_{\text {sustrato }} \mathrm{gSV}_{\text {inóculo }}{ }^{-1} \mathrm{y}$ se contó con un control negativo, que fue agua destilada más inóculo, cuyo objetivo fue realizar la corrección endógena de metano.

Análisis cinético. La producción de metano acumulado en el tiempo fue modelada, aplicando cinética de primer orden, representada con la ecuación 2 y el modelo ajustado de Gompertz, con la ecuación 3 (Donoso-Bravo et al. 2015):

$$
\begin{gathered}
B(t)=B_{0} *\left(1-\exp \left(-k_{h} * t\right)\right) \quad \text { Ecuación. 2 } \\
B(t)=P_{\text {max }} * \exp \left\{-\exp \left(\frac{R_{m * e}}{P_{\max }}(\lambda-t)+1\right)\right\} \quad \text { Ecuación. 3 }
\end{gathered}
$$

Donde, $B(t)$ es la producción de metano acumulado $\left(\mathrm{mLCH}_{4} \cdot \mathrm{gSV}^{1}\right)$; t es el tiempo en el que transcurre el experimento (d); $k_{b}$ es la constante de hidrólisis $\left(\mathrm{d}^{-1}\right)$; Bo y $\mathrm{P}_{\max }$ hacen referencia a la máxima producción de metano $\left(\mathrm{mLCH}_{4} \cdot \mathrm{gSV}^{1}\right)$ (parámetro asociado a la degradación del sustrato); $R_{n}$ es la tasa máxima de producción de metano $\left(\mathrm{mLCH}_{4} \mathrm{gSV}^{1} \cdot \mathrm{d}^{-1}\right)$; e corresponde la exponencial de 1 $(2,7183)$ y es la fase de latencia (d).

Para la estimación de los parámetros cinéticos, se usaron los datos experimentales del ensayo PBM, aplicando el algoritmo de NelderMead y usando la función Fminsearchbnd de la caja de herramientas de Matlab ${ }^{\circledR}$, para lo cual, se empleó, como criterio de optimización, la minimización de la función objetivo del error medio cuadrático. Una vez que la función de costo se ha reducido al mínimo, se determinó la inversa de la matriz de información de Fisher, con el objetivo de determinar la incertidumbre de los parámetros cinéticos, obtenidos en cada modelo (Donoso-Bravo \& Fdz-Polanco, 2013).

\section{RESULTADOS Y DISCUSIÓN}

Efecto del pretratamiento térmico sobre la producción de metano. De acuerdo con la tabla 3 , se encontró que la temperatura y el tiempo de exposición, tuvieron un efecto significativo sobre el PBM, con un $p=0,002$ y $p=0,001$, respectivamente, permitiendo maximizar la producción de $\mathrm{CH}_{4}$, con valores superiores a $150 \mathrm{mLCH}_{4} \mathrm{~g}$ $\mathrm{SV}^{1}$, asociado a una temperatura cercana a $100^{\circ} \mathrm{C}$ y un tiempo de exposición de 15 minutos, como lo muestra la figura 1. Estas condiciones son similares a las óptimas encontradas por Li et al. (2016), que fueron de $120^{\circ} \mathrm{C}$ y 15 minutos. Por otro lado, los resultados son superiores a los reportados en investigaciones con sustratos similares sin pretratamiento térmico, como las de ParraOrobio et al. (2015): 101,8mL CH4 g SV ${ }^{1}$; Cárdenas-Cleves et al. (2018): 70,9mL CH4 g SV y Sánchez-Reyes et al. (2016): $116 \mathrm{~mL}$ $\mathrm{CH}_{4} \mathrm{~g} \mathrm{SV}^{1}$.

También, se identificó que, para las temperaturas extremas a $100^{\circ} \mathrm{C}$, con un tiempo de exposición mayor a 15 minutos, la producción de $\mathrm{CH}_{4}$ decrece, fenómeno que podría estar asociado, posiblemente, a la presencia o formación de subproductos complejos difíciles de degradar, ocasionada por la reacción entre los carbohidratos, 
Tabla 3. Resultados del modelo de superficie de respuesta ajustado para potencial bioquímico de metano, alcalinidad bicarbonática, ácidos grados volátiles y $\mathrm{Ph}$.

\begin{tabular}{|c|c|c|c|c|}
\hline Componente & Coeficiente & Error estándar & Valor $\mathrm{t}$ & Valor-p \\
\hline \multicolumn{5}{|c|}{ PBM } \\
\hline Intercepto & 93,50 & 10,56 & 8,85 & $7,22 \mathrm{E}^{-09}$ \\
\hline Temperatura & 1,93 & 4,84 & 0,40 & 0,69 \\
\hline Tiempo & $-17,54$ & 4,83 & $-3,63$ & $0,001 *$ \\
\hline Interacción & 6,19 & 6.79 & 0,91 & 0,37 \\
\hline Temperatura $^{2}$ & $-23,87$ & 6,80 & $-3,51$ & $0,002 *$ \\
\hline Tiempo $^{2}$ & $-0,24$ & 6,82 & $-0,03$ & 0,97 \\
\hline \multicolumn{5}{|c|}{$\mathrm{AB}$} \\
\hline Intercepto & 402,68 & 67,13 & 6,00 & 4,07E-06 \\
\hline Temperatura & 23,56 & 30,75 & 0,77 & 0,4513 \\
\hline Tiempo & $-25,29$ & 30,71 & $-0,82$ & 0,4188 \\
\hline Interacción & 35,88 & 43,18 & 0,83 & 0,4145 \\
\hline Temperatura $^{2}$ & $-136,07$ & 43,23 & $-3,15$ & $0,0045 *$ \\
\hline Tiempo $^{2}$ & $-23,50$ & 43,36 & $-0,54$ & 0,5931 \\
\hline \multicolumn{5}{|c|}{ AGV`s } \\
\hline Intercepto & 211,92 & 28,36 & 7,47 & 1,35E-07 \\
\hline Temperatura & $-13,64$ & 12,99 & $-1,05$ & 0,3045 \\
\hline Tiempo & $-17,33$ & 12,97 & $-1,34$ & 0,1947 \\
\hline Interacción & $-46,08$ & 18,24 & $-2,53$ & $0,0189 *$ \\
\hline Temperatura $^{2}$ & 46,01 & 18,26 & 2,52 & $0,0192 *$ \\
\hline Tiempo $^{2}$ & 28,85 & 18,32 & 1,58 & 0,1288 \\
\hline \multicolumn{5}{|c|}{$\mathrm{pH}$} \\
\hline Intercepto & 7,78 & 0,17 & 46,62 & $<2 \mathrm{e}-16$ \\
\hline Temperatura & 0,04 & 0,08 & 0,48 & 0,6333 \\
\hline Tiempo & 0,04 & 0,08 & 0,57 & 0,5772 \\
\hline Interacción & 0,11 & 0,11 & 1,06 & 0,3023 \\
\hline Temperatura $^{2}$ & 0,06 & 0,11 & 0,52 & 0,6103 \\
\hline Tiempo $^{2}$ & 0,02 & 0,11 & 0,17 & 0,8660 \\
\hline
\end{tabular}
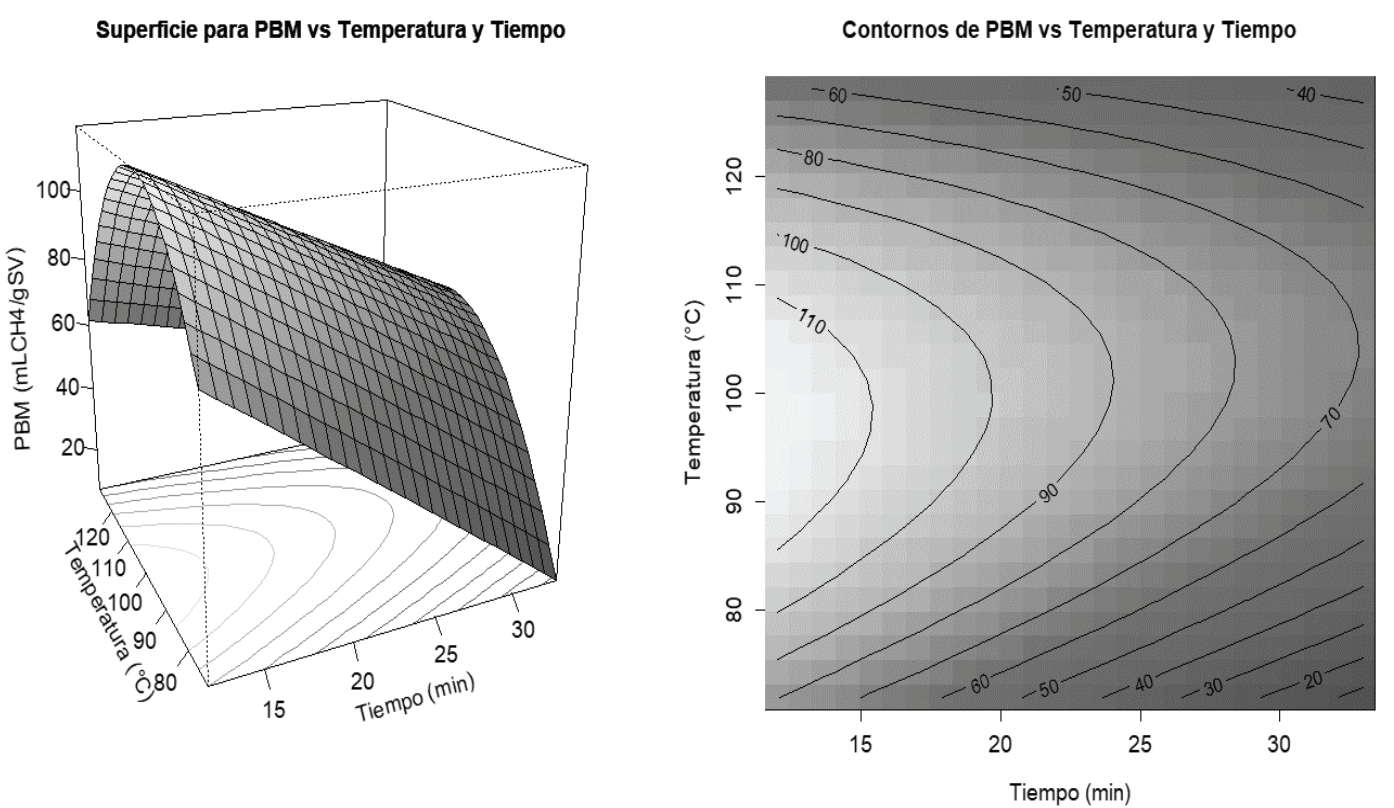

Figura 1. Superficie de respuesta para potencial bioquímico de metano vs temperatura y tiempo y gráfico de contornos para PBM vs temperatura y tiempo. 
Parra-Orobio, B.A.; Vásquez-Franco, C.; Torres-López, W.A.; Marmolejo-Rebellón, L.F.; Torres-Lozada, P.: Pretratamiento en producción de metano
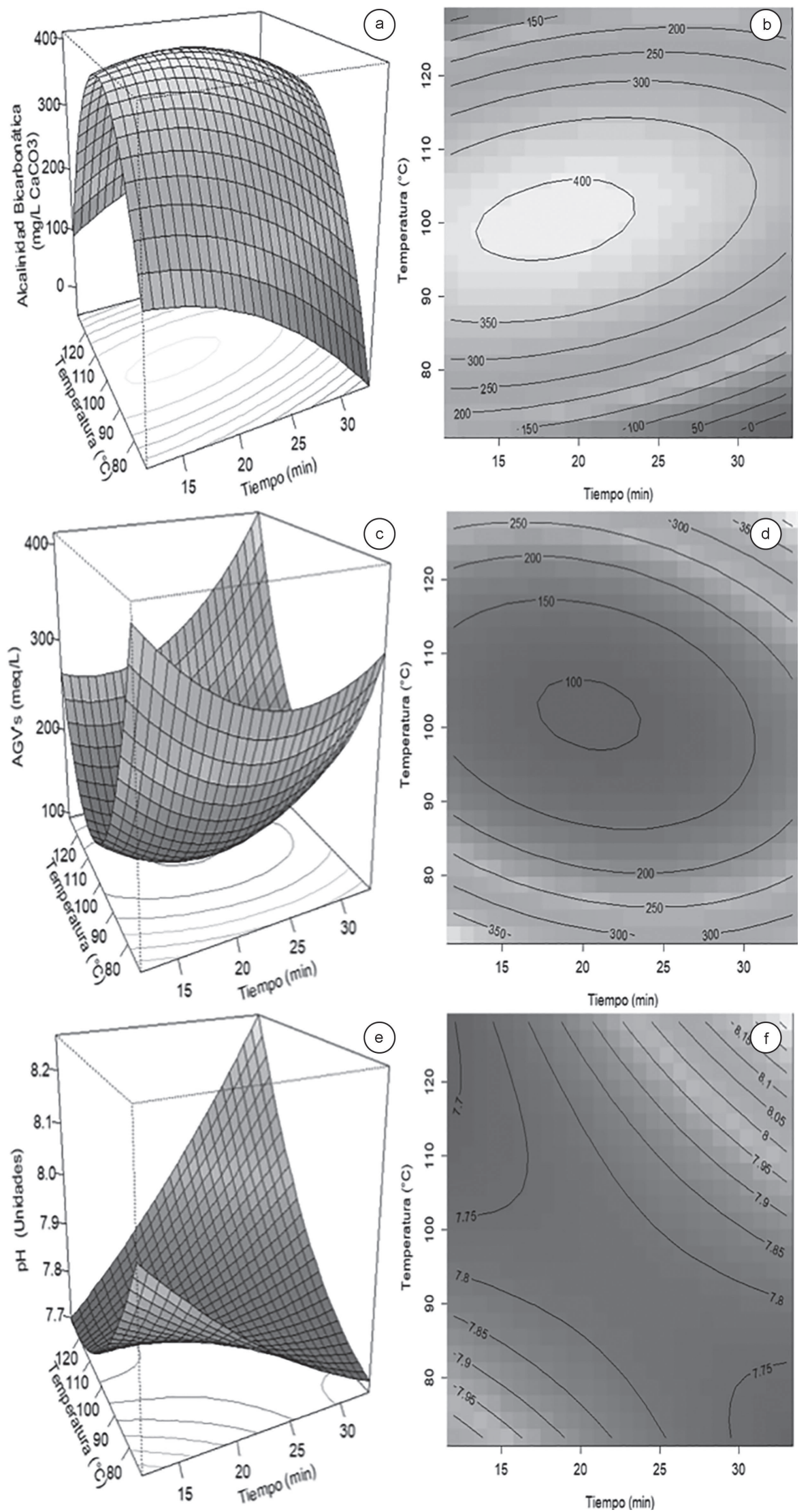

Figura 2. Alcalinidad bicarbonática-AB final vs tiempo y temperatura: a. superficie de respuesta; b. gráfico de contornos. AGV's final vs tiempo y temperatura: c. superficie de respuesta; d. gráfico de contornos. $\mathrm{pH}$ final vs tiempo y temperatura para $\mathrm{pH}$; e. superficie de respuesta; f. gráfico de contornos. 
que representan el 56\% del sustrato y los aminoácidos, reacción conocida como Mallaird (Mailard, 1916; Hendriks \& Zeeman, 2009; Ariunbaatar et al. 2014). Investigaciones relacionadas por Fujishima et al. (2000), señalan que una disminución de la producción de metano se relaciona por ausencia de carbohidratos.

Efecto del pretratamiento sobre la $\mathrm{AB}, \mathrm{AGV}^{\prime}$ s y pH. De acuerdo con la tabla 3 y la figura 2, se identificó que la temperatura es el factor que mayor efecto significativo presenta en cuanto a la maximización de la concentración de $\mathrm{AB}$, según la figura 2 a y a la reducción de los AGV's, representada en la figura $2 \mathrm{~b}$, con un $p=0,0045$ y $p=0,0003$, respectivamente. El tiempo mostró una incidencia significativa para ambos. Asimismo, se observa una relación inversa entre $A B$ y AGV`s, pues a medida que la $\mathrm{AB}$ se aleja de la región óptima de temperatura, que oscila entre los 98 y $100{ }^{\circ} \mathrm{C}$, aumenta la presencia de AGV's. Esto puede estar ligado a que a temperaturas cercanas a los $80^{\circ} \mathrm{C}$, el sustrato aún presenta elevadas concentraciones de AGV's, que ejercen una demanda de $\mathrm{AB}$, mientras que el incremento de temperatura por encima de $100^{\circ} \mathrm{C}$, en sustratos ricos en hidratos de carbono, generan sustancias recalcitrantes, que reducen la capacidad buffer del proceso (Dwyer et al. 2008; Ferreira et al. 2013).
Lo anterior es acorde con la amplia región identificada para el $\mathrm{pH}$, que corresponde a valores cercanos a la neutralidad; en ese factor, se encontró que la temperatura y el tiempo de exposición no presentaron un efecto significativo, lo que ratifica que el $\mathrm{pH}$ no debe ser considerado como un factor decisorio, en cuanto a la operación en la definición de estrategias de control de reactores anaerobios, siendo más sensible y mejor indicadora la alcalinidad bicarbonática (Parra-Orobio et al. 2014; Zhai et al. 2015).

Análisis cinético. La tabla 4 presenta los ajustes de los modelos cinéticos empleados para los tratamientos evaluados. Se destaca que, en general, ambos modelos tuvieron un buen ajuste con $\mathrm{R}^{2}>0,9$ y $\mathrm{CME}<7$. Respecto al modelo de primer orden, se encontró que, si bien hubo un incremento de la tasa de hidrólisis en los tratamientos $\mathrm{T} 1-80^{\circ} \mathrm{C}-15 \mathrm{~min}, \mathrm{~T} 2-80^{\circ} \mathrm{C}-30 \mathrm{~min}, \mathrm{~T} 3-120^{\circ} \mathrm{C}-15 \mathrm{~min}$ y $\mathrm{T} 6-72^{\circ} \mathrm{C}-22 \mathrm{~min}$ respecto al tratamiento control-T10, la producción de metano no incrementó. Este fenómeno reafirma lo mencionado previamente, donde se muestra que, a temperaturas mayores y menores a $100^{\circ} \mathrm{C}$, se generan sustancias reclacitrantes, que inhiben la acción de los consorcios microbianos, involucrados en cada una de las etapas de la DA de RA.

Tabla 4. Parámetros cinéticos para cada uno de los pretratamientos evaluados con su respectiva incertidumbre.

\begin{tabular}{|c|c|c|c|c|c|c|c|c|c|}
\hline \multirow[b]{2}{*}{$\mathbf{T}$} & \multicolumn{4}{|c|}{ Modelo de primer orden } & \multicolumn{5}{|c|}{ Modelo ajustado de Gompertz } \\
\hline & $\begin{array}{c}\text { Bo } \\
\left(\mathrm{mLCH}_{4} \cdot \mathrm{gSV}^{-1}\right)\end{array}$ & $k_{b}\left(\mathrm{~d}^{-1}\right)$ & $\mathbf{R}^{2}$ & CME & $\begin{array}{c}\mathbf{P}_{\max } \\
\left(\mathrm{mLCH}_{4} \cdot \mathrm{gSV}^{-1}\right)\end{array}$ & $\begin{array}{c}\mathbf{R}_{\max } \\
\left(\mathrm{mLCH}_{4} \cdot g S V^{-1} \mathrm{~d}^{-1}\right)\end{array}$ & $\lambda(\mathrm{d})$ & $\mathbf{R}^{2}$ & CME \\
\hline T1 & $94,9 \pm 2,7$ & $0,04 \pm 0,002$ & 0,98 & 3,4 & $88,1 \pm 2,4$ & $3,4 \pm 0,1$ & $0 \pm 0,9$ & 0,99 & 2,3 \\
\hline $\mathrm{T} 2$ & $64,1 \pm 1,1$ & $0,05 \pm 0,002$ & 0,99 & 1,6 & $60,7 \pm 2,3$ & $2,6 \pm 0,1$ & $0 \pm 1,2$ & 0,99 & 2,2 \\
\hline T3 & $83,8 \pm 1,9$ & $0,05 \pm 0,002$ & 0,99 & 2,6 & $78,1 \pm 3,2$ & $3,2 \pm 0,2$ & $0 \pm 1,3$ & 0,98 & 3,2 \\
\hline T4 & $122,8 \pm 10,7$ & $0,02 \pm 0,003$ & 0,97 & 4,2 & $98,5 \pm 7,4$ & $2,2 \pm 0,01$ & $0 \pm 2,9$ & 0,99 & 3,0 \\
\hline T5 & $116,7 \pm 7,4$ & $0,03 \pm 0,003$ & 0,97 & 4,1 & $54,0 \pm 1,2$ & $1,4 \pm 0,1$ & $0 \pm 1,18$ & 0,94 & 6,1 \\
\hline T6 & $53,6 \pm 1,9$ & $0,06 \pm 0,005$ & 0,96 & 3,4 & $49,2 \pm 0,9$ & $2,8 \pm 0,1$ & $2,1 \pm 0,6$ & 0,99 & 1,7 \\
\hline T7 & $83,8 \pm 14,5$ & $0,02 \pm 0,004$ & 0,93 & 4,5 & $59,2 \pm 5,3$ & $1,4 \pm 0,1$ & $0 \pm 3,3$ & 0,97 & 3,2 \\
\hline T8 & $200,8 \pm 11,8$ & $0,02 \pm 0,002$ & 0,99 & 4,1 & $142,8 \pm 9,2$ & $3,6 \pm 0,2$ & $0 \pm 2,4$ & 0,98 & 5,8 \\
\hline T9 & $73,5 \pm 3,9$ & $0,02 \pm 0,002$ & 0,99 & 1,5 & $92,1 \pm 9,1$ & $2,7 \pm 0,3$ & $0 \pm 3,7$ & 0,99 & 1,2 \\
\hline T10 & $107,4 \pm 7,2$ & $0,02 \pm 0,002$ & 0,98 & 2,9 & $77,4 \pm 5,2$ & $2,2 \pm 0,2$ & $1,3 \pm 2,4$ & 0,97 & 4,0 \\
\hline
\end{tabular}

El T8- $100^{\circ} \mathrm{C}-12 \mathrm{~min}$ fue el tratamiento que presentó mejor comportamiento en términos de producción de metano en todos los escenarios, con un aumento del 100\%, además de una tasa de hidrólisis igual a la del control, indicando que la transformación de la materia orgánica presente en los RA no originó material recalcitrante, que indujera la inhibición de la biomasa metanogénica. El mismo comportamiento, se presentó con el modelo de Gompertz, donde se resalta que, a una temperatura de $72^{\circ} \mathrm{C}$, se prolonga la fase de latencia cercana a los 2 días; en la condición T8 $-100^{\circ} \mathrm{C}-12 \mathrm{~min}$, también se logró la mayor tasa de producción de metano, que fue de 3,6 $\mathrm{mLCH}_{4} \cdot \mathrm{gSV}^{1} \mathrm{~d}^{-1}$, que representa un incremento del orden del $60 \%$ y una reducción notoria en la fase de latencia respecto al control.

Según Mirmasoumi et al. (2018), esto puede estar asociado a que, temperaturas menores a $90^{\circ} \mathrm{C}$, se requieren tiempos de exposición entre 30 minutos y 1 hora, para lograr que, durante el proceso, no se generen sustancias que reduzcan el $\mathrm{pH}$ y, en consecuencia, incidan sobre la producción de metano. Estudios realizados por Jin et al. (2016), también demuestran que, a temperaturas entre 120 y $140^{\circ} \mathrm{C}$, se lograron producciones de metano por encima de $400 \mathrm{mLCH}_{4} \cdot \mathrm{gSV}^{1}$, que superan los obtenidos en este estudio, con $\mathrm{T} 8-100^{\circ} \mathrm{C}-12 \mathrm{~min}$, lo que se debe a que, para alcanzar estos niveles de producción, se requieren tiempos de exposición cercanos a 50 minutos, lo cual, desde las perspectivas energética y económica, podría ser una desventaja para el establecimiento de un proceso a escala real, lo que amerita ser evaluado.

Por lo anterior, esta investigación permite concluir que el pretratamiento térmico a $100^{\circ} \mathrm{C}$ y 12 minutos es una opción que puede incrementar significativamente la producción de metano y 
reducir la fase de latencia; sin embargo, en el contexto de países en desarrollo, se debe evaluar el balance energético y el aspecto económico, para establecer ésta como una opción favorable y sostenible en la Digestión Anaerobia de Residuos de Alimentos.

Agradecimientos: A Colciencias, por la beca de doctoral del estudiante Brayan Alexis Parra-Orobio, Convocatoria 617 - 2013 Segundo corte y a la Universidad del Valle, por el soporte para la elaboración de este manuscrito. Conflicto de Interés: Certificamos la originalidad y el carácter inédito del manuscrito elaborado y revisado con la participación de todos los autores, quienes declaramos que no existe conflicto de intereses que ponga en riesgo la validez de los resultados. Financiación: Esta investigación fue financiada por la Universidad del Valle en el marco del proyecto de convocatoria interna 'Digestión anaerobia de una y dos fases de residuos de alimentos para la producción de energía renovable: comparación de la puesta en marcha, estabilidad y rendimiento del procesoCI-21006”.

\section{REFERENCIAS}

1. ANGELIDAKI, I.; ALVES, M.; BOLZONELLA, D.; BORZACCONI, L.; CAMPOS, J.L.; GUWY, A.J.; KALYUZHNYI, S.; JENICEK, P.; VAN LIER, J.B. 2009. Defining the biomethane potential (BMP) of solid organic wastes and energy crops: A proposed protocol for batch assays. Water Science and Technology. 59:927-934. https:/ / doi.org/10.2166/wst.2009.040

2. AMERICAN PUBLIC HEALTH ASSOCIATION-APHA 2005. Standard methods for examination of water and wastewater. American Water Works Association and Water Environment Federation. Washington D.C.

3. AQUINO, S.F.; CHERNICHARO, L.C.A.; FORESTI, E.; FLORENCIO, D.S.M.D.L. 2007. Metodologias para determinação da atividade metanogênica específica (AME) em lodos anaeróbios. Eng. Sanit. Ambient. 12:192-201. http:/ /dx.doi.org/10.1590/S1413-41522007000200010

4. ARIUNBAATAR, J.; PANICO, A.; ESPOSITO, G.; PIROZZI, F.; LENS, P.N.L. 2014. Pretreatment methods to enhance anaerobic digestion of organic solid waste. Applied Energy. 123:143-156. https://doi.org/10.1016/ j.apenergy.2014.02.035

5. CABEZA, I.; THOMAS, M.; VÁSQUEZ, A.; ACEVEDO, P.; HERNÁNDEZ, M. 2016. Anaerobic Co-digestion of Organic Residues from DifferentProductive Sectors in Colombia: Biomethanation Potential Assessment. Chemical Engineering Transactions. 49:385-390. https:/ / doi.org/10.3303/CET1649065

6. CÁRDENAS-CLEVES, L.M.; MARMOLEJO-REBELLÓN, L.F.; TORRES-LOZADA, P. 2018. Anaerobic co-digestion of sugarcane press mud with food waste: Effects on hydrolysis stage, methane yield and synergistic effects. Internal J. Chemical Engineering. 2018(2):1-8. https://doi. org/10.1155/2018/9351848

7. DHAMODHARAN, K.; KUMAR, V.; KALAMDHAD, A.S. 2015. Effect of different livestock dungs as inoculum on food waste anaerobic digestion and its kinetics. Bioresource Technology. 180:237-241. https://doi.org/10.1016/ j.biortech.2014.12.066

8. DONOSO-BRAVO, A.; FDZ-POLANCO, M. 2013. Anaerobic co-digestion of sewage sludge and grease trap: Assessment of enzyme addition. Process Biochemistry. 48(5):936-940. https://doi.org/10.1016/j.procbio.2013.04.005

9. DONOSO-BRAVO, A.; PÉREZ-ELVIRA, S.; FDZPOLANCO, F. 2015. Simplified mechanistic model for the two-stage anaerobic degradation of sewage sludge. Environmental Technology. (United Kingdom). 36(10):1334-1346. https://doi.org/10.1080/09593330.20 14.988186

10. DWYER, J.; STARRENBURG, D.; TAIT, S.; BARR, K.; BATSTONE, D.J.; LANT, P. 2008. Decreasing activated sludge thermal hydrolysis temperature reduces product colour, without decreasing degradability. Water Research. 42(18):4699-4709. https://doi.org/10.1016/ j.watres.2008.08.019

11. FERREIRA, L.C.; DONOSO-BRAVO, A.; NILSEN, P.J.; FDZPOLANCO, F.; PÉREZ-ELVIRA, S.I. 2013. Influence of thermal pretreatment on the biochemical methane potential of wheat straw. Bioresource Technology. 143:251-257. https:/ / doi.org/10.1016/j.biortech.2013.05.065

12. FUJISHIMA, S.; MIYAHARA, T.; NOIKE, T. 2000. Effect of moisture content on anaerobic digestion of dewatered sludge: ammonia inhibition to carbohydrate removal and methane production. Water Sci. Technol. 41(3):119-127.

13. HENDRIKS, A.; ZEEMAN, G. 2009. Pretreatments to enhance the digestibility of lignocellulosic biomass. Bioresource technology. 100(1):10-18. https://doi.org/10.1016/ j.biortech.2008.05.027

14. HOLLIGER, C.; ALVES, M.; ANDRADE, D.; ANGELIDAKI, I.; ASTALS, S; BAIER, U.; BOUGRIER, C.; BUFFIÈRE, P.; CARBALLA, M.; DE WILDE, V.; EBERTSEDER, F; FERNÁNDEZ, B.; FICARA, E.; FOTIDIS, I.; FRIGON, J.C.; FRUTEAU DE LACLOS, H.; GHASIMI, D.S.M.; HACK, G.; HARTEL, M.; HEERENKLAGE, J.; SARVARI HORVATH, I.; JENICEK, P.; KOCH, K.; KRAUTWALD, J.; LIZASOAIN, J.; LIU, J.; MOSBERGER, L.; NISTOR, M.; OECHSNER, H.; OLIVEIRA, J.V.; PATERSON, M.; 
PAUSS, A.; POMMIER, S.; PORQUEDDU, I.; RAPOSO, F.; RIBEIRO, T.; RÜSCH PFUND, F.; STRÖMBERG, S.; TORRIJOS, M.; VAN EEKERT, M.; VAN LIER, J.; WEDWITSCHKA, H.; WIERINCK, I. 2016. Towards a standardization of biomethane potential tests. Water Science and Technology. 74(11):2515-2522. https://doi. org/10.2166/wst.2016.336

15. INSTITUTO COLOMBIANO DE NORMAS TÉCNICAS Y CERTIFICACIÓN-ICONTEC 2004. Norma Técnica Colombiana 5167. Productos para la industria agrícola, productos orgánicos usados como abonos o fertilizantes y enmiendas de suelo. 32p.

16. INSTITUTO COLOMBIANO DE NORMAS TÉCNICAS Y CERTIFICACIÓN-ICONTEC 2009. Gestión ambiental. Residuos sólidos. Guía para la separación en la fuente. Guía Técnica Colombia GTC-24. Instituto Colombiano de Normas Técnicas y Certificación. Bogotá D.C. 3p.

17. IZUMI, K.; OKISHIO, Y.K.; NAGAO, N.; NIWA, C.; YAMAMOTO, S.; TODA, T. 2010. Effects of particle size on anaerobic digestion of food waste. International Biodeterioration \& Biodegradation. 64:601-608. https:// doi.org/10.1016/j.ibiod.2010.06.013

18. JIN, Y.; LI, Y.; LI, J. 2016. Influence of thermal pretreatment on physical and chemical properties of kitchen waste and the efficiency of anaerobic digestion. J. Environmental Management. 180:291-300. https://doi.org/10.1016/ j.jenvman.2016.05.047

19. KEMPEGOWDA, R.S.; SKREIBERG, Ø.; TRAN, K.Q.; SELVAM, P.V.P. 2017. Techno-economic assessment of thermal co-pretreatment and co-digestion of food wastes and sewage sludge for heat, power and biochar production. Energy Procedia. 105:1737-1742. https://doi. org/10.1016/j.egypro.2017.03.498

20. LI, Y.; JIN, Y.; LI, J.; LI, H.; YU, Z. 2016. Effects of thermal pretreatment on the biomethane yield and hydrolysis rate of kitchen waste. Applied Energy. 172:47-58. https://doi. org/10.1016/j.apenergy.2016.03.080

21. LIU, X.; WANG, W.; GAO, X.; ZHOU, Y.; SHEN, R. 2012. Effect of thermal pretreatment on the physical and chemical properties of municipal biomass waste. Waste Management. 32(2):249-255. https://doi.org/10.1016/ j.wasman.2011.09.027

22. MA, C.; LIU, J.; YE, M.; ZOU, L.; QIAN, G.; LI, Y.Y. 2018. Towards utmost bioenergy conversion efficiency of food waste: Pretreatment, co-digestion, and reactor type. Renewable and Sustainable Energy Reviews. 90:700-709. https://doi.org/10.1016/j.rser.2018.03.110
23. MAILARD, L.C. 1916. Synthesis of humus-like substances by the interaction of amino acids and reducing sugars. Ann Chim. 5:258-317.

24. MIRMASOUMI, S.; EBRAHIMI, S.; SARAY, R.K. 2018. Enhancement of biogas production from sewage sludge in a wastewater treatment plant: Evaluation of pretreatment techniques and co-digestion under mesophilic and thermophilic conditions. Energy. 157:707-717. https:// doi.org/10.1016/j.energy.2018.06.003

25. NEVES, L.; OLIVEIRA, R.; ALVES, M.M. 2006. Anaerobic co-digestion of coffee waste and sewage sludge. Waste Management. 26(2):176-181. https://doi.org/10.1016/ j.wasman.2004.12.022

26. OVIEDO-OCAÑA, E.R.; MARMOLEJO-REBELLÓN, L.F; TORRES-LOZADA, P. 2014. Influencia de la frecuencia de volteo para el control de la humedad de los sustratos en el compostaje de biorresiduos de origen municipal. Rev. Internal Contaminación Ambiental. 30:91-100.

27. PABÓN, P.C.P.; CASTAÑARES, G.; VAN LIER, J.B. 2012. An OxiTop ${ }^{\circledR}$ protocol for screening plant material for its biochemical methane potential (BMP). Water Science and Technology. 66(7):1416-1423. https://doi.org/10.2166/ wst.2012.305

28. PARRA-OROBIO, B.A.; ANGULO-MOSQUERA, L.S.; LOAIZA-GUALTERO, J.S.; TORRES-LÓPEZ, W.A.; TORRES-LOZADA, P. 2018. Inoculum mixture optimization as strategy for to improve the anaerobic digestion of food waste for the methane production. J. Environmental Chemical Engineering. 6(1):1529-1535. https://doi.org/10.1016/j.jece.2018.01.048

29. PARRA-OROBIO，B.A.; TORRES-LOZADA，P.; MARMOLEJO-REBELLÓN, L.F. 2017. Anaerobic digestion of municipal biowaste for the production of renewable energy: Effect of particle size. Brazilian J. Chemical Engineering. 34:481-491. http://dx.doi. org/10.1590/0104-6632.20170342s20150331

30. PARRA-OROBIO, B.A.; TORRES-LOZADA, P.; MARMOLEJO-REBELLÓN, L.F.; CÁRDENASCLEVES, L.M.; VÁSQUEZ-FRANCO, C.H.; TORRESLÓPEZ, W.A.; ORDÓÑEZ-ANDRADE, J.A. 2014. Influencia del $\mathrm{pH}$ sobre la digestión anaerobia de biorresiduos de origen municipal. Rev. U.D.C.A Act. \& Div. Cient. 17(2):553-562. http://dx.doi.org/10.31910/ rudca.v17.n2.2014.421

31. PARRA-OROBIO, B.A.; TORRES, L.P.; MARMOLEJO, L.F.; CÁRDENES, C.L.M.; VÁSQUEZ, F.C.; TORRES, L.W.A.; ORDONEZ, A.J.A. 2015. Efecto de la relación sustrato- 
inóculo sobre el potencial bioquímico de metano de biorresiduos de origen municipal. Ingeniería Investigación y Tecnología. 16(4):515-526.

32. PARTHIBA KARTHIKEYAN, O.; TRABLY, E.; MEHARIYA, S.; BERNET, N.; WONG, J.W.C.; CARRERE, H. 2018. Pretreatment of food waste for methane and hydrogen recovery: A review. Bioresource Technology. 249:10251039.

33. RAPOSO, F; DE LA RUBIA, M.; FERNÁNDEZ-CEGRÍ, V.; BORJA, R. 2012. Anaerobic digestion of solid organic substrates in batch mode: An overview relating to methane yields and experimental procedures. Renewable and Sustainable Energy Reviews. 16(1):861-877. https://doi. org/10.1016/j.rser.2011.09.008

34. SÁNCHEZ-REYES, C.; PATIÑO-IGLESIAS, M.E.; ALCÁNTARA-FLORES, J.L.; REYES-ORTEGA, Y.; PÉREZ-CRUZ, M.A.; ORTÍZ-MUÑOZ, E. 2016. Determinación del potencial bioquímico de metano (PBM) de residuos de frutas y verduras en hogares. Rev. Internal Contaminacion Ambiental 32(2):191-198. http://dx.doi. org/10.20937/RICA.2016.32.02.05

35. SEPÚLVEDA, J.A.M. 2016. Outlook of municipal solid waste in Bogota (Colombia). American J. Engineering and Applied Sciences. 9(3):477-483. https:/ / doi.org/10.3844/ ajeassp.2016.477.483

36. SOTO-PAZ, J.; OVIEDO-OCAÑA, E.R.; MANYOMA, P.C.; MARMOLEJO-REBELLÓN, L.F.; TORRES-LOZADA, P.; BARRENA, R.; SÁNCHEZ, A.; KOMILIS, D. 2019.
Influence of mixing ratio and turning frequency on the co-composting of biowaste with sugarcane filter cake: a mixture experimental design. Waste and Biomass. Valorization.1:1-15. https://doi.org/10.1007/s12649-01900592-2

37. SURENDRA, K.C.; TAKARA, D.; HASHIMOTO, A.G.; KHANAL, S.K. 2014. Biogas as a sustainable energy source for developing countries: Opportunities and challenges. Renewable and Sustainable Energy Reviews. 31:846-859. https://doi.org/10.1016/j.rser.2013.12.015

38. THI, N.B.D.; KUMAR, G.; LIN, C.Y. 2015. An overview of food waste management in developing countries: Current status and future perspective. J. Environmental Management 157:220-229. https://doi.org/10.1016/ j.jenvman.2015.04.022

39. TORRES, L.P.; PÉREZ, A. 2010. Actividad metanogénica específica: una herramienta de control y optimización de sistemas de tratamiento anaerobio de aguas residuales. Revista EIDENAR. (9):5-14.

40. ZHAI, N.; ZHANG, T.; YIN, D.; YANG, G.; WANG, X.; REN, G.; FENG, Y. 2015. Effect of initial $\mathrm{pH}$ on anaerobic co-digestion of kitchen waste and cow manure. Waste Management. 38:126-131. https://doi.org/10.1016/ j.wasman.2014.12.027

41. ZHANG, C.; SU, H.; BAEYENS, J.; TAN, T. 2014. Reviewing the anaerobic digestion of food waste for biogas production. Renewable and Sustainable Energy Reviews. 38:383-392. https://doi.org/10.1016/j.rser.2014.05.038 